\title{
Application of EDA Technology in Experimental Reform of Computer Organization
}

\author{
Lihua Jiang ${ }^{1, a}$ and Bing Ruan ${ }^{2, b^{*}}$ \\ ${ }^{1}$ School of Computer, Wuhan Polytechnic University, Wuhan Hubei, 430023, China \\ ${ }^{2}$ School of Computer, Wuhan Polytechnic University, Wuhan Hubei, 430023,China \\ ajianglihua@whpu.edu.cn, b29153056@qq.com \\ * The corresponding author
}

Keywords: Hardware Design Software; EDA Technology; VHDL

\begin{abstract}
This paper introduces the general idea of the experimental reform of computer organization: hardware design software, expounds that the practice teaching should take the experimental principle as the main body, and its present situation and trend, to explore the feasibility of the experimental reform and the advantages of the software of hardware design. Finally illustrates that the combination of teaching and scientific research is a contest theme.
\end{abstract}

\section{Introduction}

For the experimental mode of computer composition principle, most colleges and universities adopt the traditional verification experiment mode of " fixed function integrated circuit + connection". a large number of connection lines in the experiment not only easily lead to poor contact and connection errors, but also distract attention, which is not convenient for error investigation and ignores the exploration of experimental principle. The author has been engaged in the experimental teaching of the computer composition principle for many years, and the key to improve the experimental teaching effect of the computer composition principle is to guide the students to strengthen the understanding of the experimental principle, to improve the practical ability of the students' theory with practice, to arouse the innovation consciousness and to cultivate the spirit .Next, I will discuss on experiment hardware design's software of computer composition principle.

\section{Practice Teaching with Experimental Principle as the Main Body}

The principle of computer composition is a basic course of computer science and technology specialty. It tells the structure and working principle of computer from the viewpoint of hierarchy structure and the sequence of information input, processing and output, so that students can master the principles, parameters and using methods of logic devices and components commonly used in computer, learn the introductory knowledge in computer design and the basic composing principle of simple and complete single computer, and cultivate students' ability to master the analysis, design, development, use and maintenance of hardware system.

The main task of that experiment on the principle of computer composition is to make students have a more comprehensive and systematic understand of the computer composition on basis of the existing compute knowledge through learning, and to improve the students' basic knowledge of computer hardware and the ability of basic theory and practical operation. Especially through the practice of students, improve students' understanding of computer composition, maintenance and application skills. In practice, students should take the experimental principle as the main body, go deep into the computer CPU, to check and test the working state of the main signals and components, modify the realized design, increase their new design, and achieve the effect of "personally tasting pears". Only in this way, can we truly combine theory with practice, and ensure 
the theoretical depth and higher practicability of the teaching content of the main chapters of the course.

\section{The Present Situation and Development Trend of Organization experiment}

Many scholars and educational experts at home and abroad have been working hard to carry out the research on the teaching reform of the course of computer composition principle in computer major.

At home and abroad, many famous colleges and universities have noticed the importance of digital system in the course of "principles of composition", and tried to use ASIC to form a small scale computer system in the teaching content. However, due to the absence of abstract and highly general description tools, a small computer system often takes up a large amount of space to draw out the logic schematic diagram and its readability is poor. To do this, many textbooks introduced the early programmable logic device PAL, GAL or other development tools. However, the logic scale of PAL, Gal is not large, the description ability of its development tools is not strong, and it is still difficult to explore the problem in the computer system which is widely used at present. The hardware description language through the "Composition principle" course is still blank.

In the teaching content, we should grasp the basic knowledge of the basic composition and operation principle of a single computer. In the course of deepening the teaching of each function part of computer, the contents of the hardware system of computer and its operation principle are strengthened. In the whole process of teaching, we insist on the knowledge of hardware, and deepen the understanding of the connection and cooperation between hardware and software in the computer system. For example: mastering how to carry out word (chip selection technology), bit expansion, when the main memory formed by memory chip was used. Understand timing requirements when using semiconductor memory circuits.

In practice teaching, the "fixed function integrated circuit + wired" experiment mode is still the student's "main meal". "hardware design and software" is still blank in the practical teaching of "principle of computer composition", and the experimental board which is compatible with practical application has not been applied yet.

In order to break through this situation, some colleges and universities have set up another technical course to study EDA technology. The starting point of this research project is to run through the basic ideas and methods of EDA technology in the course of "principles of composition", in order to better grasp the basic theories and methods of computer systems, to Lay a solid foundation for the following courses to better reflect the latest level of the development of modern science and technology.

\section{Feasibility Analysis of Experimental Reform}

In accordance with the teaching principle of "science combined with ideological content", We introduced the basic ideas and basic methods of EDA(Electronic Design Automation) technology which is the latest achievements in the field into the teaching of "principles of computer composition", which is free from the constraints of traditional, classical analysis and Design methods, To inject new vitality into this traditional and important basic course of technology.

At present, as an important technical basic course of this subject, its teaching content still follows the traditional form of "digital electronic circuit". The description, analysis and design methods used are only applicable to simple logic components or local logical relations, especially in the experimental means, the traditional verification teaching mode is still adopted. which makes the teaching level of this course difficult to rise to the "system" level. As Hegel put it: "see the trees, do not see the forest". However, with the rapid progress and development of computer technology, the logical relationship between computer components and their interfaces is becoming more and more complex, the scale of integration is becoming larger and larger, and the speed is getting higher and higher. This situation leads to a series of computer hardware courses in theory and practice teaching is difficult to penetrate into the computer hardware system that has been widely used. At the same 
time, it is difficult to teach software courses, especially the system kernel level, which makes teaching difficult to adapt to the development level of the discipline.

EDA technology is the latest achievement in the field of computer composition principle development and design. It is compatible with the methods based on logical symbol diagram and VHDL (hardware description language, such as Altera's VHDL) in the description, analysis and design of computer composition. It is suitable for both very simple logic relations and complex large scale digital systems. The basic ideas and methods of EDA technology are used in the teaching of "principle of composition", which lays a solid foundation for improving the teaching level of this course:

1)The mastery of basic knowledge: Based on the intuitiveness of logical symbol graph and the abstraction of VHDL, the process from perceptual cognition to rational understanding is accelerated, which facilitates the transition from local, concrete to global, abstract level and conforms to "the knowledge accumulation and the combination of intelligent development" teaching principle.

2)The establishment of system concept: Based on the high efficiency of VHDL description, the description and simulation of large-scale composing principle is easy to be realized, which makes it possible to study and explore some problems in the hardware system of the computer which is widely used.

3)The innovation of experiment means: Based on the EDA Technology development tool software, the composing principle experiment uses the software design and the simulation technology which is identical with the actual development process, the final result can download directly to the FPGA chip verification. The advantages of this new experimental model are obvious:

The theory is closely related to practice, and what is learned is used.

It overcomes the problem that the teaching parties have been puzzled and difficult to solve in the traditional experimental mode: it is difficult to realize the computer system with a certain scale; It overcomes the problems that have plagued both sides of teaching, but difficult to solve: it is difficult to achieve a certain scale of computer systems. A large number of connecting wires are not only easy to lead to poor contact, but also to connection errors. but more importantly, it is not easy to troubleshoot errors. The experimental operation is inefficient. Therefore, the quality and quantity of information transmission and uptake are improved effectively.

"hardware design and software", which make use of the powerful online help function of computer, fully improve the utilization rate of teaching resources. There is no large number of connection wires abandoned, which is conducive to environmental protection.

\section{Hardware Design Software}

The experiment teaching runs through the computer logic part design the new theory and technology of ----EDA technology, achieves a new theory of the combination of teaching and research experimental teaching mode, introduces the hardware description language VHDL field programmable FPGA technology, effectively improve the teaching quality and efficiency, what you learn is what you use. It can reach up to improve teaching level, cultivate solid theoretical foundation and high quality talents with practical skills. There are the advantages of hardware design software:

1)The hardware and software components have the smallest configuration, which embodies the basic principles and basic knowledge of the computer hardware system, and does not overmuch involve many issues such as the completeness and rationality of system performance. This includes a compact instruction system, minimal monitoring program, simple controller design, small main memory, and simple input/output interfaces.

2)Under the guidance of the "systematic and gradual advancement" teaching principle, the experimental teaching process is organized. With the full adaptability of the development software, from logical symbols and VHDL "low-level statements" to logical modules and VHDL "high-level statements", a step-by-step development in depth, the realization of knowledge analysis, application, synthesis, and accumulation achieve new level. 
3)Design and simulation results are easily downloaded to verify the FPGA chip. In view of the openness of the download technology, the download cable and the simulation circuit test board can be easily manufactured and the cost is low. Students can design and produce according to actual needs in the curriculum design. Comprehensive use of computer resources and research on logic analyzer with USB interface contribute that teaching and research can reach a new level.

\section{Never Change the Theme of Teaching and Scientific Research}

In a word, students are the subject of the learning process. In the whole teaching process of the experiment, students should be fully reflected in the subject status of the study, and pay attention to the cultivation of students' independent learning ability and innovation ability. the basic ideas and basic methods of hardware design software are introduced into the practical teaching of "Computer Composition Principles", so as to get rid of traditionally experimental model and reach up to the best teaching effect.

\section{References}

[1] LIU Wei-dong, XU Ke. Structural Computer Composition. Beijing: Mechanical Industry Press, 2013.10.

[2] PAN Song, HUANG Ji-ye. Practical Course of EDA Technology. Beijing: Science Press, 2014.2.

[3] Geoffery M.Brown and Norman Vrana.A Computer Architecture Laboratory Course Using Programmable Logic[J].IEEE Transaction on Education Vol.38. No.2. May 2005.

[4] Alter co. APEX 20K Programmable Logic Device Family Data Sheet(Ver.5.1)[K], 2008

[5] GUO Lian-xi, WANG Mei-ni.The reform study on experiment teaching base on EDA[J]. Higher Agricultural Education,2005(3):58-59.

[6] CHEN Yuan, HUANG Xian-ying.Exploration on experimental teaching of "principle of computer organization" based on EDA technology[J].Journal of Chongqing Institute of Technology,2007,21(2):134-135.

[7] LI Li-ping,SHENG Lin-yang.Research on open educational model of technology computer composition principle experiment class based on EDA[J].Computer Education,2010(10): 58-59.

[8] QI Mei,ZHANG Peng,DONGYE Chang-lei.Design and realization of model machine based on CPLD combinational logic controller[J].Reseach and Exploration in Laboratory, 2010, 29(7):67-68.

[9] LIU Wen-ying,LI Ke-wen,JI You-fang. The study of practice teaching on computer hardware system B computer education[J].Computer Education,2008(14):63-64.

[10] David A.Patterons,John L.Hennesy.Computer Organization \& Design[M].America:Morgan Kaufmann,2004. 